\title{
An hypothesis approach to the solution of anagrams
}

\author{
GERALD A. MENDELSOHN \\ Institute of Personality Assessment and Research, University of California, Berkeley, Califormia 94720
}

\begin{abstract}
The attempts of subjects to reorganize the letters of an anagram were construed as a series of hypotheses about the correct letter order. It was predicted, consequently, that variables which reduce the number of tenable hypotheses or influence the order in which hypotheses are generated will affect problem difficulty. Five such variables, plus solution word frequency, were used to predict solution probabilities in two studies. The multiple Rs obtained were .92 and .82 and the two regression equations were effectively interchangeable. The process of anagram solution was described as entailing the retrieval of words from memory storage on the basis of letter order cues generated by the subject or, less usually, present in the anagram itself.
\end{abstract}

The process of anagram solution can be analyzed into two phases consisting, respectively, of attempts to reorganize the letters of the anagram and to retrieve the solution word from memory. In a recent paper, Mendelsohn and O'Brien (1974) argued that in the reorganization phase subjects formulate hypotheses about the correct letter order on the basis of the empirical probabilities of letter events in the language. Like previous investigators following the lead of Mayzner and Tresselt (1962; see also, Mayzner, Tresselt, \& Helbock, 1964), they assumed that the bigram is the basic unit with which the subject works in reorganizing the anagram, but in their formulation, the pool of bigrams that can be formed from the anagram constitutes a limited set of hypotheses from which the subject samples. The order in which hypotheses are formed corresponds roughly to the relative transition letter probabilities (TPs) of the bigrams in the pool, the most probable appearing first, and so on. The hypotheses are then tested by attempting to retrieve from memory a whole word which matches the partial reorganization of the anagram or by attempting to arrange the remaining letters about the hypothesized pair.

It follows from this formulation that, when a solution word consists of bigrams which are probable relative to the other (incorrect) bigrams which can be formed from the anagram, the likelihood of solution should be high and solution latency should be low. Conversely, when the bigrams of a solution word are relatively improbable, high latencies and infrequent solutions should be the case. The measure developed by Mendelsohn and O'Brien (1974) to index the relative frequencies of correct and incorrect bigrams consisted of the sum, across the correct bigrams, of the number of incorrect bigrams having higher TPs. It should be noted that the TPS were obtained from Mayzner and Tresselt's (1965) frequency counts and, thus, take word length and letter position into account.
Mendelsohn and O'Brien reported a correlation of about .75 between this measure and solution scores, thus confirming their prediction. It was found, further, that this relationship was uninfluenced by solution word frequency.

The present paper seeks to extend the hypothesis approach to anagram solution by examining characteristics of letters and words other than bigram TPs which may either constrain the number of tenable hypotheses the subject can formulate or affect the sequence in which hypotheses are likely to be formulated. Four such variables were identified as follows:

1. Total number of bigrams with frequency greater than zero. It should, in general, be the case that the fewer the tenable hypotheses (i.e., bigrams with frequency $>0$ which can be formed from the letters of the anagram), the less the problem difficulty. This variable is conceptually related to Ronning's (1965) "ruleout" factor, but is based on the bigram unit rather than on all letters of the anagram considered at once.

2. Number of vowels in the solution word. With very few exceptions, words of five or more letters containing a single vowel do not begin or end with the vowel. Consequently, in this case, otherwise tenable bigram hypotheses involving the vowel can be eliminated and only those in which the vowel occupies an interior position require consideration. The effect should be to reduce the number of tenable hypotheses and, thus, problem difficulty for one-vowel words.

3. First letter of the solution word, vowel or consonant. Although there are many words which begin with vowels, it is far more common for the first letter to be a consonant. In Mayzner and Tresselt's (1965) sample of 3,422 five-letter words, for example, $85 \%$ begin with a consonant; likewise, a vowel is less likely to occupy the first position than any other in five-letter words. Consequently, hypotheses involving a VC combination in Positions 1-2 should occur relatively late 
in the sequence of reorganization attempts. Anagrams formed from solution words beginning with a vowel should thus be more difficult to solve.

4. Key letters. Cohen (1968) found that anagrams containing one of the six most infrequent letters in the language $(J, K, Q, V, X, Z)$ were easier to solve. He reasoned that "uncommon letters, if present, reduced the number of letter groupings which were plausible" and thereby "remove more uncertainty than common letters" (p. 80). The letter "V," for example, the most frequent of the 6 letters above, can be preceded by 12 different letters and followed by only 6 , all vowels, according to the Underwood and Schulz (1960) count. In contrast, " $B$," the next most infrequent letter, can be preceded by 19 and followed by 17 letters. It is not infrequency per se which is critical but the limited number of combinatorial possibilities the letters possess. Thus, the presence of an infrequent letter provides the possibility of a maximizing strategy, i.e., if the reorganization effort begins with a bigram including an infrequent letter, the number of plausible hypotheses can be rapidly exhausted and in some cases reduced to one or two. Consequently, independent of the total number of plausible hypotheses or the relative probabilities of hypotheses, problem difficulty should be reduced in the case of a key letter.

Six solution word variables [bigram rank (BR), word frequency (WF), and the four just described] were used as predictors of anagram difficulty in two studies. In addition to zero-order correlations, multiple correlations were obtained and the results for each sample cross-validated on the other.

\section{METHOD}

\section{Study 1}

The data of the first study were drawn from Mendelsohn and O'Brien (1974). Since that paper includes a detailed description of the selection of solution words, construction of anagrams and anagram lists, subjects, and procedure, a brief summary can suffice. Thirty five-letter solution words were used, half of them low frequency (6 to 10) in the Kučera and Francis (1967) count and half high frequency $(35$ to 100$)$. There were five low-BR (5-30), five middle-BR (50-70), and five high-BR (90 and above) solution words within each frequency level. The actual selection of words within each of the six WF by BR cells was done at random. Each solution word was made into 15 anagrams, 5 of them one-move, 5 two-move, and 5 threemove. Of the one-move anagrams, one began with the first letter of the solution word, one with the second, and so forth. The same principle was applied to the two- and three-move anagrams. Fifteen sets of 30 anagrams were constructed such that, across the sets, each solution word appeared once in all of its 15 anagram orders. Within sets there were an equal number of one-, two-, and three-move anagrams and of anagrams beginning with the first, second, etc., letter of the solution words. Other precautions to prevent biasing practice effects and the confounding of order with other variables were taken in the construction of the sets.

Each set was presented individually to four subjects, two male and two female. Thus, there was a total of 60 responses for each solution word, 4 for each of the 15 anagram forms. The anagrams were typed on $3 \times 5$ in. index cards in capital letters, with a maximum solution time of $60 \mathrm{sec}$ and a 5 -sec delay between anagrams. Subjects gave their responses verbally.

It should be noted that this study was designed to fit an analysis of variance format, so that the full range of BR and WF values was not sampled, i.e., there are gaps within each distribution as well as range limitations.

\section{Study 2}

The design of the second study resembles that of the first in many ways, but important differences, particularly in procedure, exist. In describing the method, special attention will be paid to the differences.

Selection of solution words. Sixty-four five-letter solution words were selected in a way designed to provide heterogeneity along a number of dimensions. Five classes of words were established: single-vowel words, two-vowel words beginning with a consonant and with a vowel, three-vowel words beginning with a consonant and a vowel. All single-solution words, i.e., words whose letters cannot be reorganized to form another word, from the Schwartz and Olson (1968) list which could reasonably be assumed to be in the vocabulary of a college student were extracted and sorted into the five classes. Words were then selected to represent the full range of BR scores within a class (the range varying from class to class). For example, for twovowel words beginning with a consonant, one word was selected from the BR interval $1-10$, one from the interval $11-20$, and so on up to $131-140$. In three of the classes, the procedure of sampling from decades had to be approximated rather than precisely achieved because of the lack of a suitable distribution of scores. For one-vowel words, two words from each decade were selected in order to increase the size of this subsample. There were 16 one-vowel words, 24 two-vowel words, 10 beginning with a vowel, and 24 three-vowel words, 10 beginning with a vowel. One two-vowel word proved to be a doublesolution anagram and was not included in the data analyses. Eight of these words appeared in Study 1 and could thus be used to provide an estimate of the reliability of solution scores across the two studies. Despite the procedural differences described below, the rank-order correlation between solution scores in the two studies was .976 . Analyses including and excluding the eight words in Study 2 are virtually identical, so only the results for the full sample of 63 words will be reported.

Compared to Study 1 , the resulting distribution of BR scores was extended on both the lower and upper ends and is without gaps. Although no effort was made to control the distribution of WF counts, it, too, was more extended on both ends than in Study 1 (range from 1 to 130). However, in keeping with the distribution of all words in the language, the sample distribution is highly skewed, with a piling up of cases on the low-frequency end. No claim can be made that the sample of words in either study is representative of words in general. Only five-letter single-solution words were used in the initial pool; of those, words judged possibly to be unfamiliar to some college students were eliminated along with slang words and words of foreign origin, e.g., "adieu." Further, the selection process was deliberately manipulated in terms of the BR variable, which undoubtedly affected the distributions of the other variables as well. However, the samples were drawn from a broad and functionally significant population of words, obvious sources of bias were controlled through a systematic sampling procedure, and the range and variability of the continuous scores is great enough to permit a valid test of the predicated relationships.

Construction of anagrams and anagram lists. Fifteen anagrams were constructed for each solution word, following the same procedures as in Study 1. To construct the lists, the total sample of 64 words was divided into two parts, each composed of half the words in each class; the mean BR score for each of these halves was approximately equal. From each half, 15 anagram lists of 32 anagrams each were constructed, the lists 
being balanced, as in Study 1 , for number of letter moves and initial letter. Because of time constraints, each subject was given only one of the lists to solve.

Subjects and procedure. The subjects were 70 male and 50 female undergraduates enrolled in an upper division course in cognitive psychology. All the subjects were tested during a regular class hour at a point in the course prior to the presentation of any material directly relevant to anagram solution. Since sex differences were not found in Study 1, nor in previous research of this kind, the anagram lists were assigned to the subjects on a purely random basis. The subjects in Study 2 differed from those in Study 1 in two respects: They were older (juniors and seniors vs. freshmen and sophomores) and they were psychology majors. Consequently, they were probably more able academically.

The 32 anagrams in a list plus three warm-up anagrams were presented in dittoed booklet form, each anagram typed in capital letters. Instructions were given on the first page of the booklet as well as verbally, and all the subjects understood the task. The subjects had $15 \mathrm{sec}$ for each anagram and there was a pause of $5 \mathrm{sec}$ between each problem. The timing was controlled by the experimenter, who told the subjects when to begin, stop, and turn the page for the next problem. This undoubtedly made for a degree of imprecision in the timing, but given the design of the study, it could not have introduced any systematic biases. The procedure also made it infeasible to collect latency data, but Mendelsohn and O'Brien (1974) found solution frequency and latency measures to be equivalent.

The procedures in the two studies are clearly very different in mode and setting of the data collection (groups vs. individual) and time limit $(1 \mathrm{~min}$ vs. $15 \mathrm{sec})$. There are differences, too, in the samples of solution words and subjects. Thus, if comparability of findings across the two studies is obtained, there can be greater confidence placed in the generalizability of results.

Following completion of the anagram task, the subjects were asked to describe, first, how they went about solving the anagrams and, then, what made a difficult or an easy anagram. Finally, they were asked to write down any "theory" of the process of anagram solution they might have derived from their experience. In Study 1, a similar inquiry was conducted, but, since an individual administration was used, the inquiry was more detailed, particularly in regard to problem solving strategies.

Independent and dependent measures. The derivation of the BR measure was described earlier and is illustrated in detail in Mendelsohn and O'Brien (1974). The means and SDs for the two studies were 63.27 and 37.90 (Study 1) and 70.78 and 35.02 (Study 2). Word frequencies were obtained from Kučera and Francis (1967); the means and SDs were 34.37 and 30.08 (Study 1) and 31.06 and 32.59 (Study 2); Number of bigrams greater than zero (GTZERO) was obtained from the Mayzner and Tresselt (1965) count for five-tetter words. For each word there are 80 possible bigram by position entries (20 bigrams, each of which can appear in four positions). The measure consisted of the total number of these entries which exceeded zero. Number of vowels in the solution words (No.Vwl.), first letter of the solution word, vowel, or consonant (Beg.Cons.), and key letter (Key) were all scored on a 1 or 0 basis. For No.Vwl. a word with a single vowel received the score of 1 , a word with two or three vowels a score of 0 . For the other two variables, a score of 1 was assigned to words which began with a consonant and to words which contained one of the six most infrequent letters. The proportion of 1 scores in each sample was; No.Vwl., .20 and .25; Beg.Cons., . 77 and .70; and Key, .20 and .30.

Inspection of the intercorrelations of the six predictor variables indicates that WF and Key are essentially independent of the four other variables and each other. The remaining corre-
Table 1

Intercorrelations of Predictor Variablest

\begin{tabular}{lcc}
\hline \multicolumn{1}{c}{ Correlation } & Study 1 & Study 2 \\
\hline BR with GTZERO & $.496^{* *}$ & $.590^{* *}$ \\
No. Vwl. & $-.406^{*}$ & $-.301^{*}$ \\
Beg. Cons. & $-.546^{* *}$ & $-.270^{*}$ \\
GTZERO with No. Vwl. & $-.542^{* *}$ & $-.346^{* *}$ \\
Beg. Cons. & $-.423^{*}$ & -.233 \\
No. Vwl. with Beg. Cons. & .276 & $.383^{* *}$ \\
\hline
\end{tabular}

$\dagger$ No significant between-studies differences were obtained. ${ }^{*} p<.05 \quad{ }^{* *} p<.01$

lation coefficients are shown in Table 1 . It is evident that there is some redundancy among these four variables. Given their nature, that is, of course, inevitable, e.g., when the number of bigrams greater than zero is low, the number of bigrams whose frequencies can exceed those of the correct bigrams is reduced. However, none of the correlation coefficients is sufficiently high to permit an assumption of equivalence between any two variables. Consequently, all six were used in deriving multiple regression equations.

The dependent variable was the number of correct solutions summed across the 15 anagrams formed from a solution word. Since the time limit in Study 2 was $15 \mathrm{sec}$, for purposes of comparability, the data in Study 1 were scored for number of solutions in $15 \mathrm{sec}$, as well as $60 \mathrm{sec}$. In addition, scores for onetwo-, and three-move anagrams were obtained and results for these variables will be included when relevant.

It was predicted that bigram rank and number of bigrams greater than zero would be negatively related and the remaining variables positively related to the number of correct solutions.

\section{RESULTS}

The zero-order product-moment correlations between the six predictors and solution scores are shown in Table 2. The prediction for each variable was confirmed by the data, though in Study 1 the coefficients for WF and Key letter were not significant at the .05 level. For no variable was a significant difference between Study 1 and Study 2 obtained. In Study 1 , the data for 60-sec solution scores were quite similar to those shown in Table 2; the correlation coefficients were -.734 , $.267,-.596, .554, .685$, and .274 , respectively. In fact, all results for the $15-\mathrm{sec}$ and $60-\mathrm{sec}$ data are so similar that there is no point in reporting the latter any further.

Table 2

Correlations Between Predictors and Solution Scores

\begin{tabular}{|c|c|c|}
\hline Predictor & Study 1 & Study 2 \\
\hline $\mathrm{BR}$ & $-.750 * *$ & $-.600^{* *}$ \\
\hline WF & .311 & $.307^{*}$ \\
\hline GTZERO & $-.642 * *$ & $-.626 * *$ \\
\hline No. Vwl. & $.595^{* *}$ & $.454^{* *}$ \\
\hline Beg. Cons. & $.612^{* *}$ & $.490 * *$ \\
\hline Key & .234 & $.283^{*}$ \\
\hline
\end{tabular}


Table 3

Beta Coefficients and Estimates of Contribution for Each Predictor Variable

\begin{tabular}{|c|c|c|c|c|c|c|}
\hline \multirow[b]{2}{*}{ Variable } & \multicolumn{2}{|c|}{ Beta } & \multicolumn{2}{|c|}{ Beta $r$} & \multicolumn{2}{|c|}{ Reduction in $\mathrm{R}^{2}$} \\
\hline & I & II & I & II & I & II \\
\hline BR & $-.44 * * *$ & $-.25 * *$ & .33 & .15 & $.11^{* * *}$ & $.04 * *$ \\
\hline WF & $.20^{* *}$ & $.26 * * *$ & .06 & .08 & $.04 * *$ & $.06 * * *$ \\
\hline GTZERO & $-.20^{*}$ & $-.25 * *$ & .13 & .16 & $.02 *$ & $.04 * *$ \\
\hline No.Vwl. & $.25 * *$ & $.16^{*}$ & .15 & .08 & $.04 * *$ & $.02 *$ \\
\hline Beg.Cons. & $.21 * *$ & $.29 * * *$ & .13 & .14 & $.03 *$ & $.07 * * *$ \\
\hline Key & $.19^{*}$ & $.24 * * *$ & .04 & .07 & $.03^{*}$ & $.05^{* * *}$ \\
\hline $\mathrm{R}^{2}$ & & & .84 & .68 & & \\
\hline
\end{tabular}

Multiple regression equations were then obtained for both studies and the multiple correlation coefficients calculated. The equations were

$\begin{aligned} \text { Study } 1 & -.146 \text { BR }+.086 \text { WF }-.354 \text { GTZERO } \\ & +7.742 \text { No.Vwl. }+6.294 \text { Beg. Cons. }+5.787 \\ & \text { Key }+37.770\end{aligned}$

Study $2-.087$ BR +.097 WF -.368 GTZERO +4.538 No. Vwl. + 7.659 Beg.Cons. + 6.371 Key +37.722 .

The multiple Rs were $.915(\mathrm{df}=6 / 23, \mathrm{p}<.001)$ and $.822(\mathrm{df}=6 / 56, \mathrm{p}<.001)$, respectively. When the equation from Study 2 was used to predict solution scores in Study 1 , the correlation coefficient between predicted and obtained scores was .899. The corresponding correlation coefficient when the equation of Study 1 was applied to 2 was .810 . In short, the two equations are virtually interchangeable.

To indicate the relative importance to prediction of each of the independent variables, the standard partial regression coefficients (beta coefficients) and their significance levels are shown in Table 3 . In addition, an estimate of the contribution of each variable to the total predicted variance of solution scores suggested by Guilford and Fruchter (1973), the beta coefficient $X$ the coefficient of correlation with the dependent measure, is included. These estimates are presented solely to facilitate comparison between the results of Study 1 and Study 2. They should not be used to draw general conclusions about which variable is the most and which the least important contributor to the solution process, for, as Darlington (1968) has shown, the assessment of the independent contributions of predictors to the multiple regression is a difficult, if not insoluble, problem when they are intercorrelated.

In light of the previous results, it is not surprising that the beta coefficients and estimates of contribution are quite similar in the two studies. There is one noteworthy exception, however: BR is a far more important predictor in Study 1 than in Study 2. The difference in total predicted variance, $84 \%$ vs. $68 \%$, seems attributable primarily to the lower correlation of BR with solution scores in the second study (see Table 2). It can also be observed in Table 3 that the four variables introduced in this research, GTZERO, No.Vwl., Beg.Cons., and Key, contribute substantially to the prediction of solution scores. A comparison of the squared multiple Rs based on BR and WF alone with those based on all six predictors provides a direct measure of the added contribution of the four variables as a set. In Study 1 the $R^{2}$ s were .646 and .838 , respectively $[F(4,23)=6.81, p<.001$ for the difference], and in Study 2 they were .384 and .677 , respectively $[F(4,56)=12.70, p<.001]$. Finally, the last two columns in Table 3 show the reduction in the proportion of predicted variance that resulted when each variable was removed singly from the set of predictors, i.e., the tabled value is the $R^{2}$ obtained from all six predictors minus the $R^{2}$ obtained with the predictor in question removed. This value provides a measure of the contribution to prediction of each variable which cannot be accounted for by any other variable or set of variables. With few exceptions, the values are expectably small, but they represent in every case at least a marginally significant gain in prediction and, for each of the six variables, $\mathrm{p}<.05$ in at least one of the studies. It seems clear, then, that despite some redundancy among the predictor variables, each has an independent effect on the solution process.

Since the dependent variable in these regression analyses was the number of correct solutions summed across the 15 anagrams formed from a solution word, the effect of anagram variables on solution scores was not evaluated. It has been shown in previous research that one such variable, number of letter moves, is reliably and strongly related to anagram difficulty (Dominowski, 1966; Mendelsohn \& O'Brien, 1974). To assess the effects of the letter moves variable and to compare its contribution to prediction with that of the solution word variables, a words by letter moves analysis of variance was performed on the data of each study. In both, the letter moves effect was highly significant: in Study 1, F $(2,58)=69.95, \mathrm{p}<.001, \mathrm{MS}_{\text {residual }}=$ 5.67 and in Study 2, $F(2,124)=124.79, \mathrm{p}<.001$, $\mathrm{MS}_{\text {residual }}=4.58$. The percentages of correct responses for one-, two-, and three-move anagrams were 62,34 , and 27 and 63,41 , and 35 , respectively. It is worth noting that, contrary to the finding of Dominowski (1966), a higher percentage of two- than three-move anagrams was solved in both studies. Since the same relationship holds in three additional samples of anagram data presently being analyzed, it appears that some particularity of design accounts for Dominowski's surprising finding.

The final step in the analysis of variance was to partition the total sums of squares ( SS $\left._{\text {total }}\right)$ into independent between-words, within-words (letter moves), and residual components. The proportion of $\mathrm{SS}_{\text {total }}$ accounted for by the between-words component was 
.58 in Study 1 and .64 in Study 2 . Given the $R^{2} s$ of .84 and .68 , this means that .49 and .44 of the $\mathrm{SS}_{\text {total }}$ was attributable to the multiple regression in the two studies. Letter moves accounted for .30 (Study 1) and .24 (Study 2) of the $\mathrm{SS}_{\text {to tal }}$ and the proportion assigned to residual variation was .12 in both studies. It can be observed that the solution word variables as a set provided the most substantial prediction of solution scores, though letter moves are clearly an important determinant of anagram difficulty.

\section{DISCUSSION}

The hypothesis approach which guided this research was outlined in the introduction, but it is important to emphasize that fundamentally the solution of anagrams is a retrieval problem, i.e., the subject's task is to retrieve a word which is already available in memory. Mendelsohn and O'Brien (1974) suggested that the retrieval process is associative in nature, with the partially reorganized anagram serving as a stimulus which evoked a whole-word response. The likelihood that the correct word will be evoked depends upon two factors: word frequency and the similarity between the reorganized anagram and the solution word. The negative relationship between the former and anagram difficulty has been well established in prior, as well as in the present, research and requires no further comment here. The latter represents the distinctive focus of the present research. In effect, the reorganization phase can be interpreted as a series of attempts to attain a letter order which matches at least partially that of the solution word. As argued earlier, the attempts are guided by the subject's knowledge of the probabilities of letter events in the language. The present data show that transition letter probabilities constitute only one aspect of that knowledge, for the additional variables introduced in this research also have predictable effects on solution scores. It seems likely, in fact, that the earlier emphasis on bigram TP has been excessive. There may be additional variables as well which affect the sequence of reorganization attempts, e.g., consonant-vowel patterns, and there is preliminary evidence that some subjects adopt different strategies depending on the number of vowels in the anagram. Nevertheless, it is evident that the set of variables identified in this pair of studies has strong and replicable effects on the solution of anagrams.

Now, a caution is necessary at this point: Although the regression equations, and the bivariate relationships they reflect, were remarkably stable across studies, they apply specifically to five-letter anagrams. Undoubtedly, equations obtained for anagrams of other lengths would be different, at least in their constants. But the data of Kaplan and Carvellas (1968) suggest further that, for anagrams of more than six letters, units of reorganization in addition to the bigram, particularly suffixes, are of significance and, consequently, that variables indexing such units should be included in the set of predictors. To conclude that the letter infor- mation employed or emphasized varies as a function of word length need not imply that the process of anagram solution itself also varies with word length, however. Indeed, the Kaplan and Carvellas (1968) study serves to strengthen the view that the solution process entails systematic efforts to reorganize the anagram as given and that the empirical probabilities of letter events in the language are the basis of these efforts. Nevertheless, the generalizability of the specific conclusions of the present pair of studies to anagrams of varying lengths must remain open to question until the necessary empirical work has been done.

The particular focus of this research was on solution word variables which influence the subject's attempts to reorganize the anagram, but the description of the solution process has implications for anagram variables as well. That is, those anagrams which have letter sequences corresponding to those in the solution word should be easier to solve. The finding that the proportion of anagrams solved is substantially higher for onethan for two- or three-move anagrams can be attributed, then, to the decreasing similarity of the anagram to the solution word as one goes from one- to three-move anagrams. However, once the effect of letter moves is partialled out, the residual variation among anagram forms for a given solution word is small. In Study 1, the SD of residuals was greater than 1.00 for only 6 words and the median SD was .83; in Study 2, 14 words had an SD $>1.00$ and median SD was .86. Considering that a substantial portion of the residual variation is attributable to differences among subjects in anagram solving ability, it is safe to conclude that the specific arrangement of the letters in the anagram, apart from letter moves, has in general little effect on problem difficulty.

It should be noted that this account of the anagram solution process is quite similar to that given by Dominowski, Johnson, and Rothwell (Note 1), who describe anagram solving "as similar to retrieving words from long-term storage." However, they conclude from their results that the dominant solution process consists of "trying to think of possible solution words based on minimal changes in the anagram as presented," and so stress the importance of word frequency and cues to solution contained within the anagram. Though they acknowledge that subjects may generate their own cues (by rearranging the letters), this is viewed as a distinctly secondary process. This position differs markedly from that of most other investigators and, in particular, from the description of the solution process derived from the results of the present studies. It also differs from the description of the process given by subjects. Seventy percent of subjects reported one form or another of a letter rearrangement procedure based on letter event or letter sequence information as their primary strategy. (It is of interest that each of the variables employed in this study was noted spontaneously by at least several subjects.) Another 20\% reported using such a strategy after attempts to go directly from the anagram to the 
solution word failed. The remainder used some form of algorithm, a pronouncing strategy, or tried to think of the solution word on the basis of the anagram as given (fewer than $5 \%$ ). Virtually all subjects reported that, on some anagrams, they simply "saw" or "flashed on" the solution. It is clear in these reports, then, that the dominant solution process involves a systematic attempt to reorganize the letters of the anagram but that. in some cases. especially one-move anagrams, the solution word may be directly evoked by the anagram upon or shortly after its presentation (see Mendelsohn $\&$ O'Brien, 1974, p. 573 for data relevant to this point). In such cases, reorganization of the anagram would, of course, be obviated.

The difference in viewpoint between Dominowski et al. (Note 1) and the present paper is probably accounted for by the many differences in procedure and variables employed in the two studies. The most im. portant of these are that: (1) Dominowski et al. used only one anagram form for each solution word; (2) all solution words began with a consonant and were selected from extremes of the WF distribution; and (3) the solution word variables in the two studies were entirely different. Given their design and variables, it is not surprising that Dominowski et al. minimized the role of solution word variables and, consequently, selfgenerated cues in the solution process. Despite the differences, however, the conclusion that the solution process consists of the retrieval of whole words from memory storage, where the retrieval is based on the similarity of part cues to the whole, seems justified by the available data. In some instances, the order of letters in the anagram as given will provide sufficient cues for retrieval, but, more generally, cues are generated by the subject in the course of systematic reorganization efforts based on empirical probabilities of letter events in the language. Studies of anagram solution, then, are relevant not only to the understanding of verbal problem solving, but also to processes of retrieval from long-term storage and the use of stored frequency information in this process.

\section{REFERENCE NOTE}

1. Dominouski. R. L.. John, D. \& Rothwell. W. Characteristics determining anagram difficulty. Paper presented at the 11th meeting of the Psychonomic Society. November 1970.

\section{REFERENCES}

Conen. J. L. The effect of letter frequency on anagram solution times. Psychonomic Science. 1968. 11. 79-80.

Darington, R. B. Multiple regression in psychological research and practice. Psychological Bulletin. 1968.69. $161-182$.

Dommowsk. R. L. Anagram solving as a function of letter moves. Journal of Verbal Learning and Verbal Behavior. 1966. 5. 107-111.

Guilford. J. P.. \& Fruchter. B. Fundamental statistics in psychology and education (5th ed.). New York: McGrawHill. 1973.

Kaplan. I. T., \& Carvelias. T. Effect of word length on anagram solution time. Joumal of Verbal Learning and Verbal Behavior. 1968. 7. 201-206.

Kúcera. H.. \& Francis, W. Computational analysis of present-day American English. Providence. R.I: Brown University Press. 1967.

Mayzner. M. S.. \& Tresselt, M. E. Anagram solution times: A function of wond transition probabilities. Journal of Experimental Psychology. 1962. 63. 510-513.

Mayzner, M. S., \& Tresselt. M. E. Tables of single-letter and bigram frequency counts for various word-length and letter-position combinations. Psychonomic Monognaph Supplements. 1965, 1, 13-31.

Mayzerer. M. S.. Tresseit, M. E.. \& Helbock. H. An exploratory study of mediational responses in anagram problem solving. Journal of Psychology, 1964. 57. 263-274.

Mendelsoms, G. A., \& O'BRIEN, A. T. The solution of anagrams: $A$ reexamination of the effects of transition letter probabilities. letter moves. and word frequency on anagram difficulty. Memory and Cognition. 1974. 2. 566-574.

Ronniwg. R. R. Anagram solution times: A function of the "ruleout" factor. Joumal of Experimental Psychology. 1965. 69. 35-39.

SCHWARTz. R.. \& Olson, R. Are alleged single-solution anagrams really single-solution? Joumal of Verbal Leaming and Verbal Behavior. 1968, 1, 567-568.

UNDERTOOD, B. J., \& SCHULz. R. W. Meaningfulness and verbal learming. Philadelphia: Lippincott. 1960.

(Received for publication December 8. 1975: revision accepted March 15. 1976.) 